\title{
Group A streptococcal toxic shock syndrome developing in the third trimester of pregnancy
}

\author{
Nancy F. Crum ${ }^{1}$, Helen M. Chun ${ }^{1}$, Thomas G. Gaylord ${ }^{2}$ and Braden R. Hale ${ }^{1}$ \\ ${ }^{1}$ Internal Medicine Department, Infectious Diseases Division and \\ ${ }^{2}$ Obstetrics and Gynecology Department, Naval Medical Center San Diego, San Diego, CA 92134
}

\begin{abstract}
Background: Group A streptococcal (GAS) toxic shock syndrome (TSS) is an uncommon, but life-threatening infection during pregnancy and should be considered in rapid onset of shock. Most cases described in the literature have occurred in the puerperium. We report a case of GAS TSS occurring during the third trimester of pregnancy in a previously healthy woman.

Case: A 31 -year-old female, who was 34 weeks pregnant, presented with fevers and a prodromal 'flu-like' illness. She rapidly developed shock and multiorgan failure. Blood cultures revealed GAS bacteremia and the patient met criteria for streptococcal TSS. Despite her eventual recovery, her infant died on postpartum day I5 as a consequence of the mother's TSS.

Conclusions: This case is unusual in that there were no identifiable initiating events or source of the streptococcal infection, and the TSS developed during pregnancy rather than after delivery. Early recognition of GAS infections is important given the rapid onset and high morbidity and mortality associated with these infections. This is the first reported case utilizing intravenous immunoglobulin for GAS TSS in the puerperium.
\end{abstract}

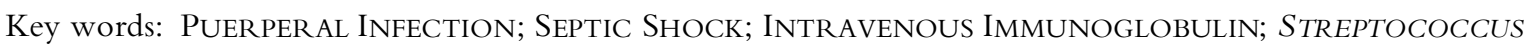
PYOGENES

Puerperal group A streptococcal (GAS) infections were well known prior to the $20^{\text {th }}$ century and referred to as 'childbed fever'. The relatively high prevalence of these infections in obstetric teaching institutions was the basis for the landmark epidemiologic investigation by Ignaz Semmelweis advocating handwashing within hospitals, a practice that dramatically decreased the occurrence of these infections ${ }^{1,2}$. Although both invasive and pregnancy-associated GAS infections were uncommon during most of the $20^{\text {th }}$ century, over the past two decades an increasing number of these infections have been described ${ }^{3-6}$. This trend may be related to the increasing prevalence of strains with specific M-proteins (M-1 and M-3). Despite antibiotics and supportive therapy, the mortality of toxic shock syndrome (TSS) due to GAS remains high $(30 \%)^{7}$. We present a case of GAS TSS in a pregnant woman in her third trimester of pregnancy, which resulted in the infant's death.

\section{CASE REPORT}

A 31-year-old woman, gravida 2 , para $0-0-1-0$, who was 34 weeks pregnant, presented to her obstetrician with an 18-hour history of fevers, chills, and suprapubic and lower back pain. She reported no notable medical problems except for a 5-day history of upper respiratory symptoms and myalgias. Her pregnancy was uneventful prior to

Correspondence to: N. F. Crum, MD, c/o Clinical Investigation Department (KCA), Naval Medical Center San Diego, 34800 Bob Wilson Drive, Suite 5, San Diego, CA 92134-1005, USA. Email: nfcrum@nmcsd.med.navy.mil

The views expressed in this article are those of the authors and do not reflect the official policy or position of the Department of the Navy, Department of Defense, or the United States Government. 
these symptoms. On presentation, she was illappearing with a temperature of $38.6^{\circ} \mathrm{C}$, pulse of $127 \mathrm{beats} / \mathrm{minute}$ and blood pressure of 104/64 mmHg. Physical findings included diffuse lower abdominal pain with mild rebound tenderness and uterine tenderness. The cervix was dilated $1 \mathrm{~cm}$ with no effacement, no evidence of ruptured membranes and no discharge. The skin and mucosal surfaces were unremarkable and without abrasions; there was no rash. Her chest and throat exams were unrevealing. The patient was suspected of having chorioamnionitis and was admitted to the labor and delivery unit. On admission, blood cultures, vaginal culture, urinalysis, and urine culture were performed before the initiation of antibiotics. Laboratory values on admission included a white blood cell count of $9700 / \mathrm{mm}^{3}$, hematocrit of 34\%, platelet count of $124000 / \mathrm{mm}^{3}$, and differential of $93 \%$ segmented neutrophils and $5 \%$ band forms. The patient was empirically treated with gentamicin, clindamycin, and vancomycin, as she reported a penicillin allergy. Despite antibiotics and intravenous fluids, the patient continued to have increasing lower abdominal pain. External fetal monitoring revealed periodic pulse decelerations to 90 beats/ minute. An obstetric ultrasound showed a fetus with an estimated weight of $2216 \mathrm{~g}$ and adequate fluid. Further decelerations were noted to 60 beats/minute prompting an emergency Cesarean section. The fetus had Apgar scores of $0 / 0$ and was pulseless for 20 minutes during the resuscitation. The infant was admitted to the neonatal intensive care unit in critical condition on ventilatory and circulatory support.

Following the Cesarean section, the mother developed respiratory insufficiency requiring intubation. A chest radiograph revealed diffuse pulmonary infiltrates and bilateral pleural effusions consistent with acute respiratory distress syndrome. Within 4 hours of the Cesarean section, bleeding at the wound site was noted as well as hypotension. The patient was rushed to the operating room where a hemoperitoneum was discovered. The patient was noted to have disseminated intravascular coagulation (DIC) with a platelet count of $54000 / \mathrm{mm}^{3}$, fibrinogen of $61 \mathrm{mg} / \mathrm{dl}$, prothrombin time of 19.7 seconds, INR 1.9, partial thrombin time 73.9 seconds, and more than 20 fibrin split products. During the following 24 hours, the patient required 11 units of packed red blood cells, 19 units of fresh frozen plasma and 36 units of platelets. The patient remained hypotensive despite fluids and packed RBC transfusions requiring vasopressor support with dopamine and phenylephrine.

On hospital day 2, two blood cultures from admission were positive for GAS, sensitive to penicillin, clindamycin, erythromycin, and vancomycin. In addition, the placenta was noted to have abundant Gram-positive cocci in pairs and chains on histopathology. Tissue culture of the placenta was positive for GAS. The urinalysis was unremarkable, and urine and vaginal cultures were negative. Because the patient remained in septic shock, intravenous immunoglobulin (IVIG) at $150 \mathrm{mg} / \mathrm{kg}$ daily for a 5-day duration and intravenous clindamycin and ceftriaxone were administered for the diagnosis of streptococcal TSS. No skin or other lesions were noted on repeated examinations. A throat culture was negative for GAS. Repeated postoperative exams and ultrasound evaluations failed to reveal ongoing pelvic infection. The infant was empirically treated with ampicillin and cefotaxime; all cultures from the infant remained negative.

Also on hospital day 2, the mother continued to have abdominal distension and was returned to the operating room where 11 of intraperitoneal blood was evacuated. Further bleeding near the right fallopian tube was noted, and a right salpingectomy was performed. The uterus appeared atonic, but there was no evidence of necrosis or active infection. The patient remained on ventilatory support, with a peak end expiratory pressure (PEEP) of 15 and $\mathrm{paO}_{2}$ of $70-100 \%$, and vasopressor support.

On day 3, the patient developed oliguric renal failure requiring continuous veno-venous hemodialysis (CVVHD). In addition, liver dysfunction was noted with an alanine aminotransrase (ALT) of $92 \mathrm{units} / \mathrm{l}$, total bilirubin of $8.2 \mathrm{mg} / \mathrm{dl}$, and direct bilirubin of $5.4 \mathrm{mg} / \mathrm{dl}$. On hospital day 4, an exploratory laparotomy was performed revealing a myometrial hematoma without necrosis. During this procedure a feeding jejunostomy tube and a gastric tube were placed for nutritional support. Due to persistent thrombocytopenia despite resolution of the DIC, ceftriaxone was discontinued on 
hospital day 5. The patient remained in critical condition over the next week, but no further major surgical intervention was required.

On day 11, a cholecystomy drain was placed due to persistent biliary sludging with a total bilirubin of $19.3 \mathrm{mg} / \mathrm{dl}$. On day 12, the patient developed a rash, and antibiotics were changed to cefotaxime and levofloxacin, which she continued to complete a 14-day course. The patient was extubated after 19 days of ventilatory support. On hospital day 25, hemodialysis was discontinued, and the patient was discharged from the hospital. Her laboratory values subsequently normalized with a new baseline creatinine level of $1.4 \mathrm{mg} / \mathrm{dl}$. The patient remains in good health and has returned to work.

Despite aggressive supportive care, the infant died on postpartum day 15 with stage III hypoxic brain damage due to asphyxia at delivery, grade IV intraventricular hemorrhage, hydrocephalus, and brainstem herniation. There was no evidence of infection in the infant.

\section{DISCUSSION}

This case emphasizes that clinicians should consider GAS in life-threatening infections occurring during pregnancy. Our patient's presentation with high fevers and chills after a 'viral-like' prodrome is characteristic of GAS infections. GAS pelvic infections may have a paucity of symptoms or signs related to the genital tract with non-specific symptoms or gastrointestinal manifestations predominating ${ }^{8}$. Our patient's fulminant clinical course demonstrates several features of streptococcal TSS, including the rapid onset of acute respiratory distress syndrome, septic shock requiring vasopressors, oliguric renal failure, liver dysfunction, and disseminated intravascular coagulation. The criteria for the diagnosis of streptococcal TSS have been previously published ${ }^{9}$. The pathophysiology of streptococcal TSS is related to the production of pyrogenic exotoxins, particularly exotoxin A, an exotoxin closely related to the Staphylococcus endotoxin responsible for staphylococcal TSS ${ }^{10}$. Exotoxin A, a superantigen, can directly stimulate $\mathrm{T}$-cells to release massive amounts of inflammatory cytokines resulting in rapid clinical sequelae.
The primary site of infection or introduction of the invasive streptococci is typically the skin, throat, or vagina. In up to $45 \%$ of cases, the primary site may be unapparent ${ }^{7}$. In most described cases of puerperal GAS infections, organisms ascend the genital canal, and the infection occurs shortly after completion of the pregnancy. Silver reported two cases occurring 2-14 days after normal vaginal deliveries ${ }^{11}$. Another case occurred after a spontaneous abortion, while other cases of GAS infection have been linked to vaginal or abdominal hysterectomy, Cesarean section, genital tract abscesses, and intrauterine contraceptive devices ${ }^{12}$. Definite cases of GAS TSS in the intrapartum or puerperium reported since 1990 in the English literature located by a MEDLINE search (using the terms 'group A streptococci', 'Streptococcus pyogenes', 'shock', 'pregnancy', and 'puerperal') are shown in Table $1^{11-25}$. Most GAS puerperal cases occur sporadically with the patients' endogenous flora; however, outbreaks have been reported due to nosocomial spread and require prompt isolation and identification of the source of the infection $^{26-29}$.

Recently, a case of streptococcal TSS has been described after vaginal delivery in which GAS was isolated during routine surveillance cultures performed to detect group B streptococci (GBS), suggesting that colonization may be a subsequent risk factor for invasive disease ${ }^{20}$. The same authors also presented a second case of GAS endometritis occurring in a patient with a positive surveillance culture. One recent study showed that vaginal or rectal colonization with GAS during pregnancy is rare, with a prevalence of $0.03 \%$, suggesting that screening specifically for GAS is not warranted ${ }^{30}$. Studies on the usefulness of treating GAS colonization found incidentally during 35- to 37-week screens in pregnancy are needed ${ }^{31}$. Our patient developed invasive disease before surveillance cultures for GBS were collected; however, vaginal cultures for streptococci were negative on admission.

In contrast to puerperal infections that typically arise from colonization or infection of the genital tract, most cases of streptococcal TSS in nonpregnant adults have a primary site of infection in the soft tissues or skin $^{3}$. Cases of streptococcal necrotizing fasciitis of the extremities occurring 


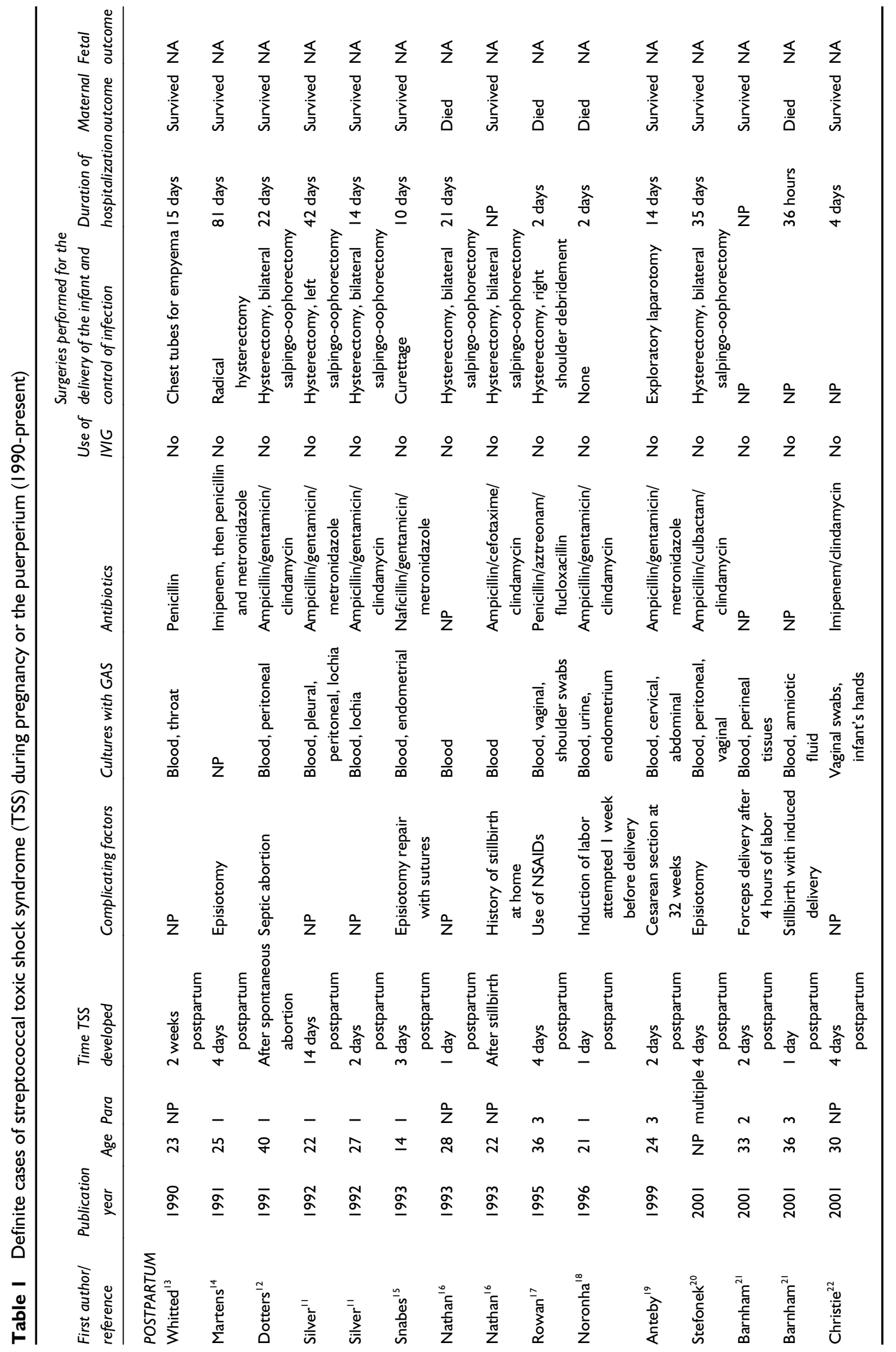




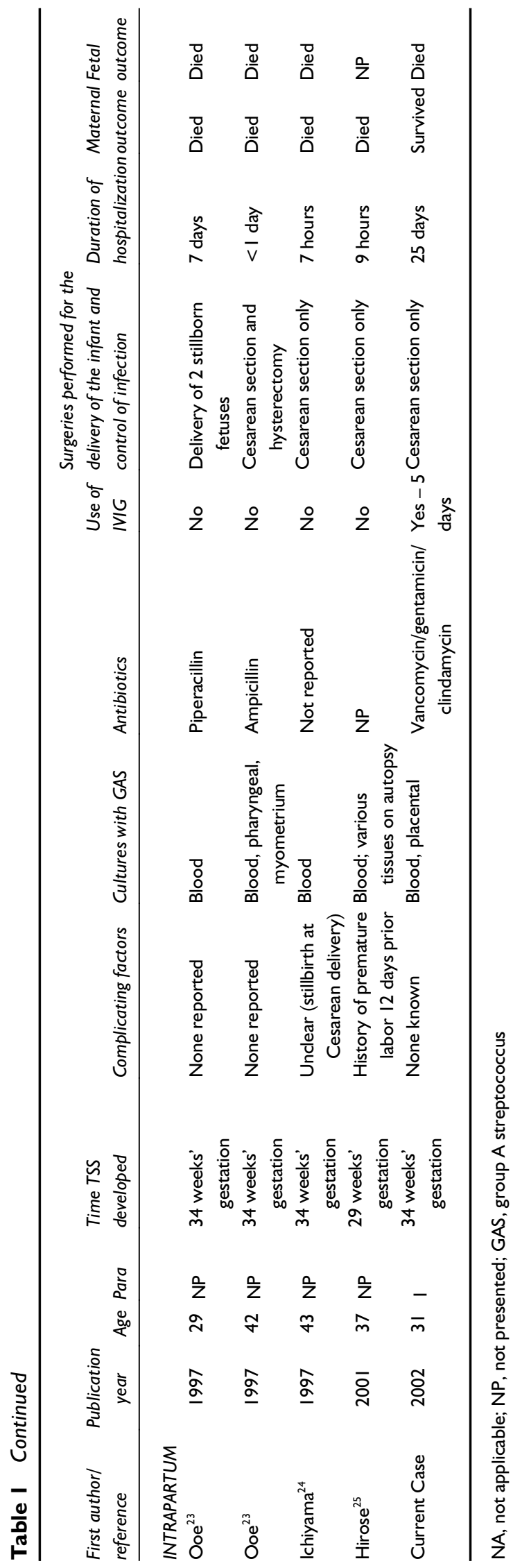

INFECTIOUS DISEASES IN OBSTETRICS AND GYNECOLOGY • 213 
during pregnancy or postpartum have been reported ${ }^{17,32}$, but the genital tract is typically the source for infections in pregnancy. Other cases in non-pregnant adults have been linked to GAS pneumonia, meningitis, peritonitis, pharyngitis, endophthalmitis, and joint infection ${ }^{33}$.

In our case, the source of the bacteremia was unclear. We were suspicious of a genital tract source because: (1) our patient was in her third trimester of pregnancy; (2) she presented with lower abdominal pain; and (3) there was no evidence for infection of the skin or pharynx. Placental histopathology and cultures revealed GAS, but it is unclear if the placenta was seeded from maternal bacteremia or if it was primarily infected. There was no evidence of vaginal infection or involvement of the uterus after delivery. This case is unusual in that sepsis developed before delivery and the source of the infection was unclear. Four additional intrapartum cases were found in our literature search ${ }^{23-25}$. In all these cases, a pharyngeal source for the GAS was proven or strongly suspected. Four of five cases, including our case, developed at 34 weeks' gestation during a previously unremarkable pregnancy. In all four other cases, the mother and fetus died when the outcome was noted. A small case series from Japan showed that TSS developing before or within 12 hours of delivery, when compared with puerperal cases, have a higher rate of both fetal and maternal mortality ${ }^{34}$.

Cases of GAS infection associated with pregnancy have often required hysterectomy. The adnexa may be preserved if active infection in these sites is absent ${ }^{11}$. In cases in which the uterus is clearly involved, hysterectomy is generally indicated. In our case, it was debated whether a hysterectomy was indicated since necrotizing streptococcal infections typically require debridement of the involved tissues. Repeated radiologic and intraoperative examinations of the uterus did not reveal necrosis, abscess or other signs of active infection. Therefore, the patient was treated without hysterectomy and subsequently recovered.

In the treatment of GAS infections, antibiotic therapy should include a $\beta$-lactam agent and clindamycin. Clindamycin is advocated because of its effectiveness despite a high inoculum of bacteria (the 'Eagle effect') and its reduction of toxin production by inhibition of protein synthesis $^{7,35}$. The use of IVIG has been shown to reduce mortality in cases of invasive GAS infection that are complicated by shock. Case-control studies show a reduction of mortality from 67 to $34 \%{ }^{36}$. Although a randomized clinical trial has never been conducted, most experts advocate the use of IVIG - preferably early in the disease course - for patients with shock due to invasive GAS infections ${ }^{37-39}$.

This case represents the first report utilizing IVIG in GAS TSS occurring in the puerperium and reminds clinicians to include invasive GAS in the differential diagnosis of a puerperal infection when a patient presents with the rapid onset of septic shock. Most postpartum cases result from ascending genital tract infection, whereas cases developing intrapartum may have a pharyngeal or occult primary source. GAS TSS occurring in association with pregnancy has a high morbidity and mortality, especially in cases occurring intrapartum.

\section{REFERENCES}

1. Adriaanse AH, Pel M, Bleker OP. Semmelweis: the combat against puerperal fever. Eur J Obstet Gynecol Reprod Biol 2000;90:153-8

2. Gilbert GL. Group A streptococci and puerperal fever. Med J Aust 1985;143:271-2

3. Stevens DL, Tanner MH, Winship J, et al. Severe group A streptococcal infections associated with a toxic shock-like syndrome and scarlet fever toxin A. N Engl J Med 1989;321:1-7
4. Gaworzewska E, Colman G. Changes in the pattern of infection caused by streptococcus pyogenes. Epidemiol Infect 1988;100:257-69

5. Ispahani P, Donald FE, Aveline AJD. Streptococcus pyogenes bacteremia: an old enemy subdued, but not defeated. J Infect 1988;16:37-46

6. Stollerman GH. Changing group A streptococci: the reappearance of streptococcal 'toxic shock'. Arch Intern Med 1988;148:1268-70 
7. Stevens DL. Invasive group A streptococcus infections. Clin Infect Dis 1992;14:2-13

8. Ledger WJ, Headington JT. Group A $\beta$-hemolytic streptococcus: an important cause of serious infections in obstetrics and gynecology. Obstet Gynecol 1972;39:474-82

9. The Working Group on Severe Streptococcal Infections. Defining the group A streptococcal toxic shock syndrome: rationale and consensus definition. J Am Med Assoc 1993;269:390-1

10. Johnson LP, L'Italien JJ, Schlievert PM. Streptococcal pyrogenic exotoxin type A (scarlet fever toxin) is related to Staphylococcus aureus enterotoxin B. Mol Gen Genet 1989;203:354

11. Silver RM, Heddleston LN, McGregor A, Gibbs RS. Life-threatening puerperal infection due to group A streptococci. Obstet Gynecol 1992;79: 894-6

12. Dotters DJ, Katz VL. Streptococcal toxic shock associated with septic abortion. Obstet Gynecol 1991;78:549-51

13. Whitted RW, Yeomans ER, Hankins GDV. Group A $\beta$-hemolytic streptococcus as a cause of toxic shock syndrome: a case report. J Reprod Med 1990;35:558-60

14. Martens PR, Mullie A, Goessens L. A near-fatal case of puerperal sepsis. Anaesth Intensive Care 1991;19:108-10

15. Snabes MC, Martens MG. A severe puerperal group A streptococcal infection causing a toxic shock-like syndrome. Int J Gynecol Obstet 1993; 40:245-8

16. Nathan L, Peters MT, Ahmed AM, Leveno KJ. The return of life-threatening puerperal sepsis caused by group A streptococci. Am J Obstet Gynecol 1993;169:571-2

17. Rowan JA, North RA. Necrotizing fasciitis in the puerperium. Am J Obstet Gynecol 1995;173:241-2

18. NoronhaS, Yue C-T, Sekosan M. Puerperal group A beta-hemolytic streptococcal toxic-shock like syndrome. Obstet Gynecol 1996;88:728

19. Anteby EY, Yagel S, Hanoch J, et al. Puerperal and intrapartum group A streptococcal infection. Infect Dis Obstet Gynecol 1999;7:276-82

20. Stefonek KR, Maerz LL, Nielsen MP, et al. Group A streptococcal puerperal sepsis preceded by positive surveillance cultures. Obstet Gynecol 2001; 98:846-8

21. Barnham MRD, Weightman NC. Bacteraemic Streptococcus pyogenes infection in the peripartum period: now a rare disease and prior carriage by the patient may be important. J Infect 2001;43:173-6
22. Christie JH, Keay SD, Morgan M, Storley R. Puerperal sepsis: a disease of the past? $\mathrm{Br} \mathrm{J} \mathrm{Obstet}$ Gynaecol 2001;108:126-32

23. Ooe K, Udagawa H. A new type of fulminant group A streptococcal infection in obstetric patients: report of two cases. Hum Pathol 1997;28: 509-12

24. Ichiyama S, Nakashima K, Shimokata K, et al. Transmission of Streptococcus pyogenes causing toxic shock-like syndrome among family members and confirmation by DNA macrorestriction analysis. J Infect Dis 1997;175:723-6

25. Hirose Y, Shibuya H, Okazaki E, et al. Toxic shock-like syndrome with flu-like prodrome: a possible role of 'enhancing tissue focus' for streptococcal toxic shock. J Infect 2001;42:195-200

26. Gordon G, Dale BAS, Lochhead D. An outbreak of group A haemolytic streptococcal puerperal sepsis spread by the communal use of bidets. $\mathrm{Br} J$ Obstet Gynecol 1994;101:447-8

27. Meis JF, Muytjens HL, van den Berg PP, et al. Analysis of an outbreak of puerperal fever due to group A streptococci by random amplified polymorphic DNA fingerprinting. Infect Dis Obstet Gynecol 1997;5:232-6

28. Ejlertsen T, Prag J, Pettersson E, Holmskov A. A 7-month outbreak of relapsing postpartum group A streptococcal infecitons linked to a nurse with atopic dermatitis. Scand J Infect Dis 2001;33:734-7

29. Centers for Disease Control and Prevention. Nosocomial group A streptococcal infections associated with asymptomatic health-care workers - Maryland and California, 1997. Morb Mortal Week Rev 1999;48:163-6

30. Mead PB, Winn WC. Vaginal-rectal colonization with group A streptococci in late pregnancy. Infect Dis Obstet Gynecol 2000;8:217-19

31. Mead PB. Streptococcal screening in obstetrics. Obstet Gynecol 2001;98:721-2

32. McHenry CR, Azar T, Ramahi AJ, Collins PL. Monomicrobial necrotizing fasciitis complicating pregnancy and puerperium. Obstet Gynecol 1996; $87: 823-6$

33. Bisno AL, Stevens DL. Streptococcus pyogenes (including streptococcal toxic shock syndrome and necrotizing fasciitis). In: Mandell, Douglas, and Bennett's Principles and Practice of Infectious Diseases, 5th edn. Philadelphia: Churchill Livingstone, 2000;2101-17

34. Udagawa H, Oshio Y, Shimizu Y. Serious group A streptococcal infection around delivery. Obstet Gynecol 1999;94:153-7 
35. Eagle H. Experimental approach to the problem of treatment failure with penicillin. I. Group A streptococcal infection in mice. Am J Med 1952; 13:389-99

36. Kaul R, McGeer A, Norrby-Teglund A, et al. Intravenous immunoglobulin therapy for streptococcal toxic shock syndrome: a comparative observation study. Clin Infect Dis 1999;28:800-7

37. Perez CM, Kubak BM, Cryer HG, et al. Adjunctive treatment of streptococcal toxic shock

ReCEIVED 04/23/02; ACCEPTED 09/03/02 syndrome using intravenous immunoglobulin: case report and review. Am J Med 1997;102:111-13

38. Lamothe F, D'Amico P, Ghosn P, et al. Clinical usefulness of intravenous human immunoglobulins in invasive group A streptococcal infections: case report and review. Clin Infect Dis 1995;21:1469-70

39. Cawley MJ, Briggs M, Haith LR, et al. Intravenous immunoglobulin as adjunctive treatment for streptococcal toxic shock syndrome associated with necrotizing fasciitis: case report and review. Pharmacother 1999;19:1094-8 


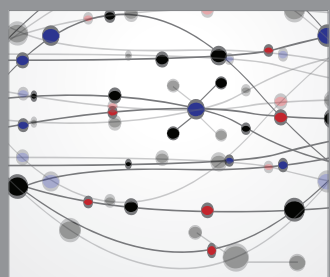

The Scientific World Journal
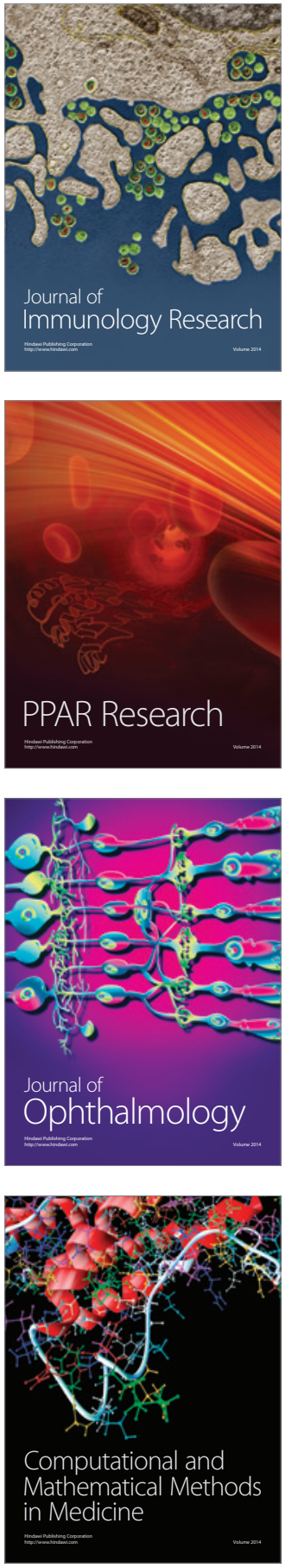

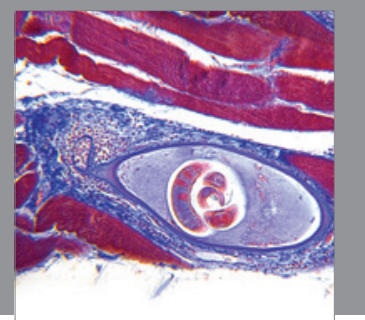

Gastroenterology

Research and Practice
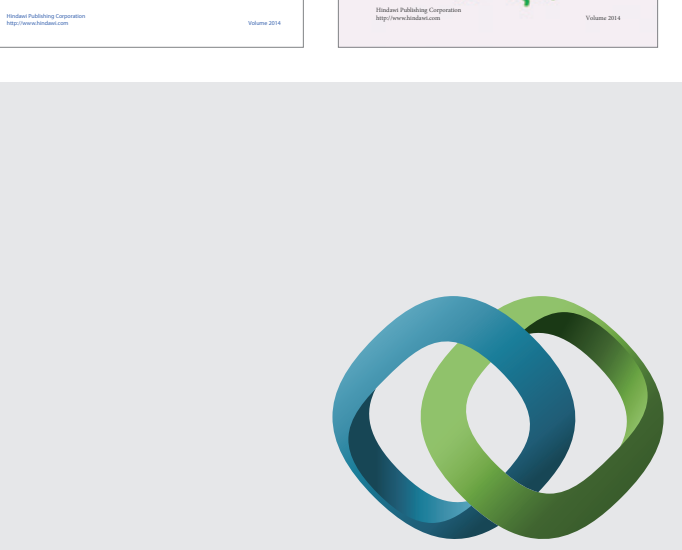

\section{Hindawi}

Submit your manuscripts at

http://www.hindawi.com
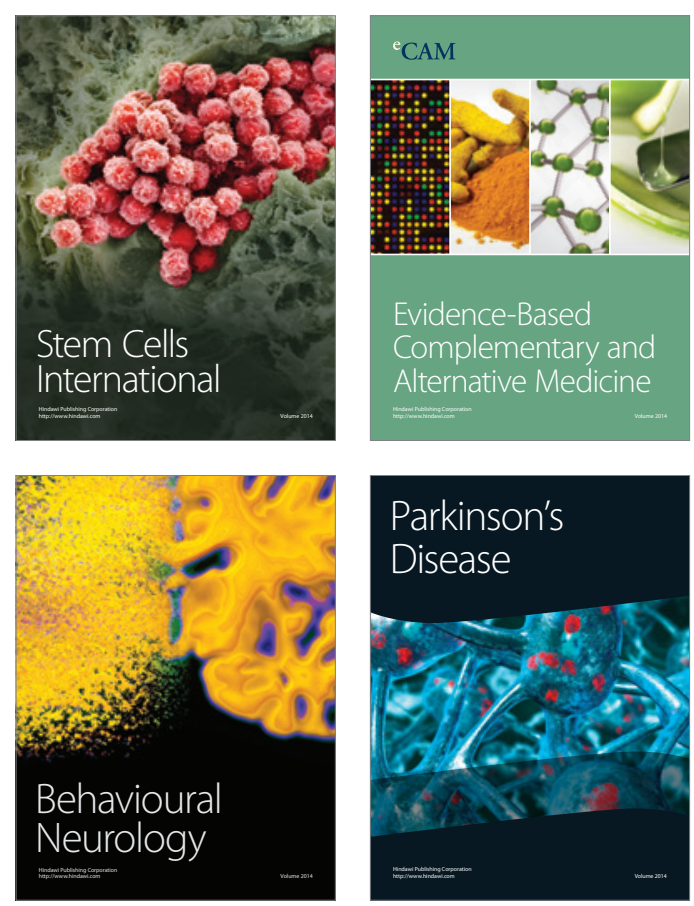

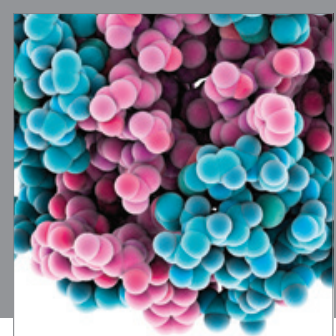

Journal of
Diabetes Research

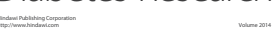

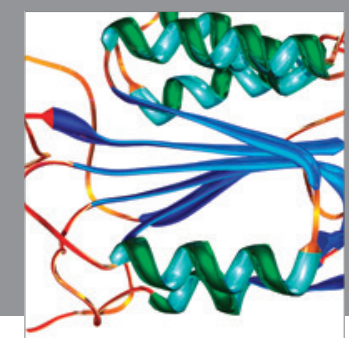

Disease Markers
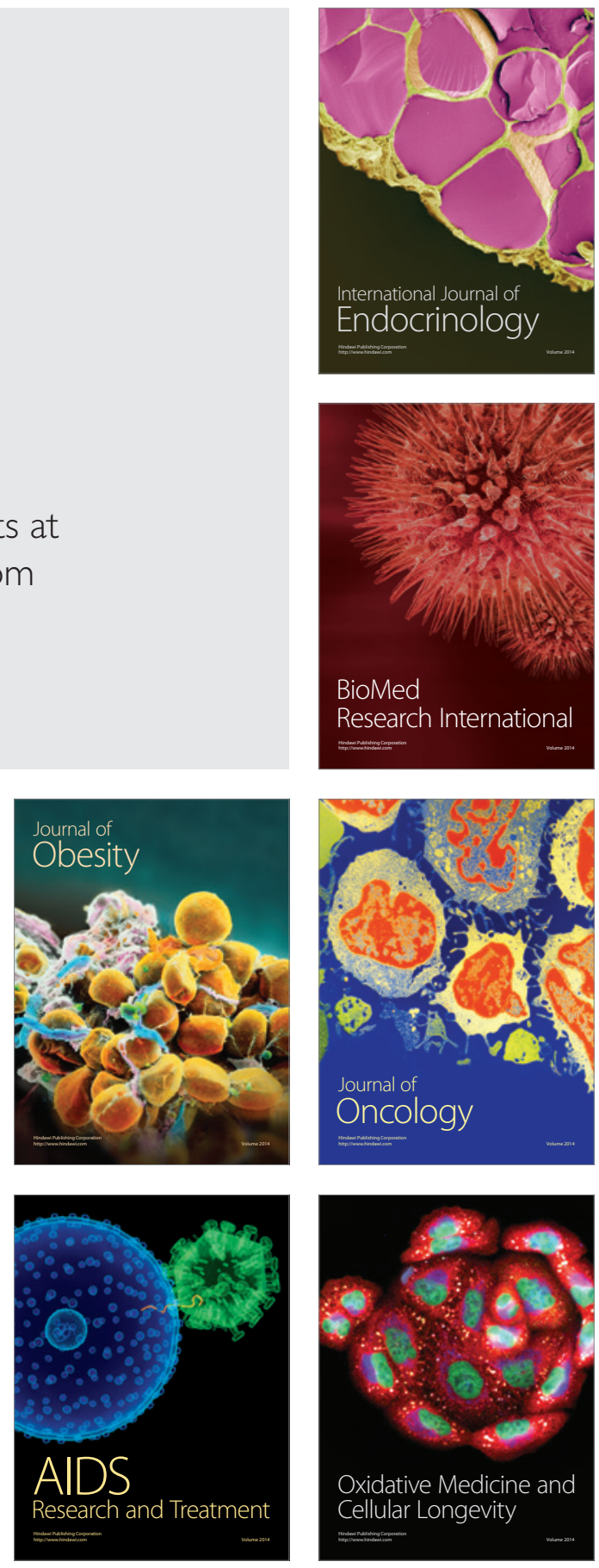